\title{
Characteristics of intracerebral haemorrhage associated with COVID-19: a systematic review and pooled analysis of individual patient and aggregate data
}

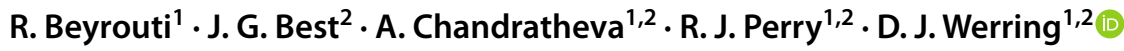

Received: 21 December 2020 / Revised: 18 January 2021 / Accepted: 22 January 2021 / Published online: 5 February 2021

(c) The Author(s) 2021

\begin{abstract}
Background and purpose There are very few studies of the characteristics and causes of ICH in COVID-19, yet such data are essential to guide clinicians in clinical management, including challenging anticoagulation decisions. We aimed to describe the characteristics of spontaneous symptomatic intracerebral haemorrhage (ICH) associated with COVID-19.

Methods We systematically searched PubMed, Embase and the Cochrane Central Database for data from patients with SARS-CoV-2 detected prior to or within 7 days after symptomatic ICH. We did a pooled analysis of individual patient data, then combined data from this pooled analysis with aggregate-level data.

Results We included data from 139 patients (98 with individual data and 41 with aggregate-level data). In our pooled individual data analysis, the median age (IQR) was 60 (53-67) years and 64\% (95\% CI 54-73.7\%) were male; 79\% (95\% CI 70.0-86.9\%) had critically severe COVID-19. The pooled prevalence of lobar ICH was 67\% (95\% CI 56.3-76.0\%), and of multifocal ICH was 36\% (95\% CI 26.4-47.0\%). 71\% (95\% CI 61.0-80.4\%) of patients were treated with anticoagulation (58\% (95\% CI 48-67.8\%) therapeutic). The median NIHSS was 28 (IQR 15-28); mortality was 54\% (95\% CI 43.7-64.2\%). Our combined analysis of individual and aggregate data showed similar findings. The pooled incidence of ICH across 12 cohort studies of inpatients with COVID-19 ( $n=63,390)$ was $0.38 \%$ (95\% CI $0.22-0.58 \%$ ).

Conclusions Our data suggest that ICH associated with COVID-19 has different characteristics compared to ICH not associated with COVID-19, including frequent lobar location and multifocality, a high rate of anticoagulation, and high mortality. These observations suggest different underlying mechanisms of ICH in COVID-19 with potential implications for clinical treatment and trials.
\end{abstract}

Keywords COVID-19 $\cdot$ Intracerebral haemorrhage $\cdot$ Stroke $\cdot$ Anticoagulation

\section{Introduction}

Coronavirus Disease 2019 (COVID-19) can be complicated by a coagulopathy with a high risk of serious and often life-threatening thromboembolic events including

D. J. Werring

d.werring@ucl.ac.uk

1 Comprehensive Stroke Service, National Hospital for Neurology and Neurosurgery, University College Hospitals NHS Foundation Trust, Queen Square, London WC1N 3BG, UK

2 Stroke Research Centre, Department of Brain Repair and Rehabilitation, UCL Queen Square Institute of Neurology, First Floor, Russell Square House, 10-12 Russell Square, London WC1B 5EH, UK venous thromboembolism [1,2] and ischaemic stroke [3]. This has led to the widespread use of anticoagulants including heparins, often guided by D-dimer levels as evidence for the degree of hypercoagulability [4]. While anticoagulants appear to improve outcome in severe COVID-19 [5], they also have the potential to increase the risk of serious intracranial bleeding, particularly intracerebral haemorrhage (ICH).

There are a few data available on the clinical features of ICH in people with COVID-19 [6, 7]. Previous reports did not allow for clear conclusions due to small sample sizes. Information about the characteristics and causes of ICH in COVID-19 is essential to guide clinicians in optimising clinical management, including making difficult anticoagulation decisions. We, therefore, undertook a systematic review and pooled analysis of all available studies reporting individual 
or aggregate-level patient data on $\mathrm{ICH}$ in the context of COVID-19.

\section{Methods}

\section{Search strategy and study selection}

We carried out a systematic literature search from PubMed, Embase and Cochrane Central Database, according to Preferred Reporting Items for Systematic Reviews and Meta-Analyses (PRISMA) guidelines [8], with the following search terms (("stroke" OR "cerebrovascular" OR "intracranial" OR “intracerebral" OR "intraparenchymal”) AND ("haemorrh*" OR "hemorrh*")) AND ("COVID-19" OR "coronavirus" OR "corona virus" OR "Coronavirus" OR "2019-nCoV” OR "SARS-CoV” OR "Severe Acute Respiratory Syndrome"). We limited the results to 2019-2020 to exclude papers referring to other coronaviruses. We did not limit the search by language. We used EndNote software to run auto de-duplication. After removal of duplicates, two reviewers (RB and JB) performed an initial search and screening for relevant articles through title and abstract; discrepancies were resolved by consensus, and where necessary with a third senior reviewer (DJW). The potential full texts were evaluated by applying the inclusion and exclusion criteria given below. The literature search was finalized on December 12, 2020.

\section{Inclusion and exclusion criteria}

We included all studies that reported patients confirmed to have severe acute respiratory syndrome coronavirus 2 (SARS-CoV-2) infection according to the WHO interim guideline [9] (i.e. a nasopharyngeal or oropharyngeal swab positive for SARS-CoV-2 on reverse transcriptase polymerase chain reaction (RT-PCR), or a blood sample positive for SARS-CoV-2 antibodies) with SARS-CoV-2 detected either prior to, at the time of, or up to 7 days post, spontaneous (nontraumatic) symptomatic ICH. We excluded studies reporting other types of intracranial haemorrhage including microhaemorrhages, ischaemic infarcts with haemorrhagic transformation, subdural hematoma, subarachnoid haemorrhage and haemorrhagic leukoencephalopathy. Review articles, editorials, opinion papers and guidelines were excluded. For the pooled individual patient data analysis, we included papers that presented individual-level data on any of our variables of interest. Papers presenting aggregate-level data only were subsequently included in a pooled analysis of both individual patient and aggregate data.

\section{Data extraction}

The following variables were extracted from the studies: demographics (age, gender); vascular risk factors; ICH characteristics (location, cause); stroke severity (National Institutes of Health Stroke Scale (NIHSS) score); anticoagulant treatment (prophylactic or therapeutic) at the time of ICH; COVID-19 severity and clinical outcome; coagulation and inflammation markers (D-dimers and fibrinogen, APPT, PT, INR, platelets, CRP, ferritin). We classified ICH from reported information according to location as lobar or non-lobar (i.e. deep or infratentorial, defined as ICH in the basal ganglia, thalamus, brainstem or the cerebellum). We then classified ICH aetiology using all information available by predefined criteria as follows: hypertensive arteriopathy (deep or infratentorial haemorrhage with known hypertension before the ICH); cerebral amyloid angiopathy (defined as $\geq 1$ lobar, cortical, or cortico-subcortical haemorrhage and age $\geq 55$ with no other identified cause); structural macrovascular lesions (aneurysm, pseudoaneurysm, cavernous malformation); coagulopathy (defined as $\mathrm{PT} \geq 15$ and/or INR $\geq 1.2$ and/or APTT $\geq 45$ and/or platelets $\leq 100,000$ ); other identified causes (cerebral venous thrombosis, Moya Moya angiopathy); or undetermined.

To estimate the pooled incidence of ICH in patients with COVID-19, we extracted data on the number of hospitalised patients with COVID-19 and the number documented to have ICH in the same time period.

\section{Statistical analysis}

A pooled analysis was performed on all of the individual patient data found in the published literature, to estimate means, medians, standard deviations, minimum and maximum values. We then combined the aggregate data from these individual patients with other studies reporting aggregate data according to the two-stage method [10], using a random-effects model. We compared the characteristics of patients who were and were not receiving anticoagulation at the time of their ICH using Fisher's exact test. Statistical analyses were performed using Microsoft Office Excel version 36516.0 and Stata 16.

\section{Results}

\section{Literature search and screening}

The flow diagram (Fig. 1) shows the detailed literature search steps and data selection for our individual patient data pooled analysis. The database searches identified 


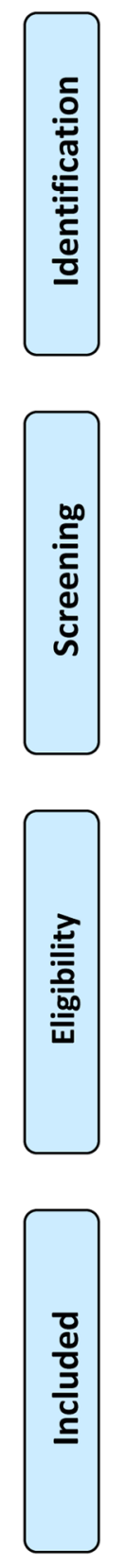

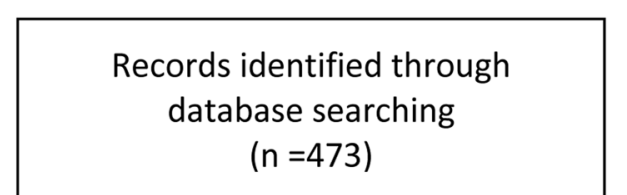

Additional records identified through manual search of relevant article references

$$
(n=6)
$$

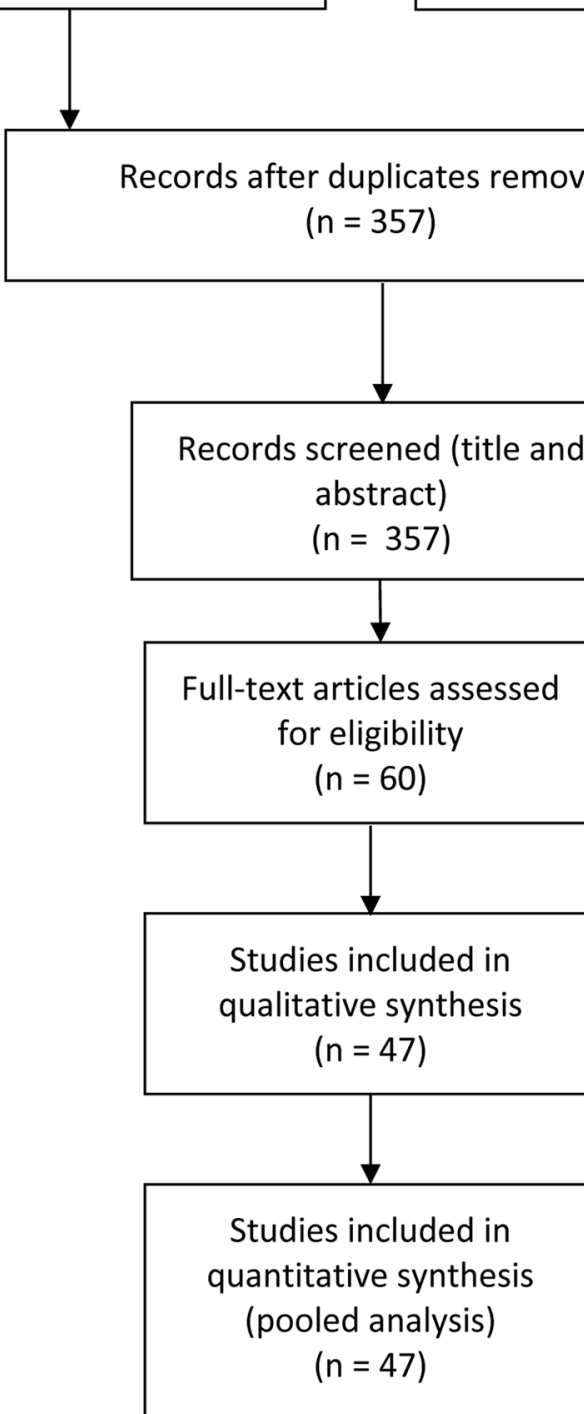

Records excluded, with reasons:

review articles, editorials,

opinion papers and guidelines $(n=297)$

ull-text articles excluded, with reasons:

presented aggregate-level data only

$(n=13)$

Fig. 1 Flow diagram of the literature search and selection process in the pooled individual patient data analysis

a total of 473 potentially relevant articles. Six further articles were added through a manual search of relevant article references. After the exclusion of duplicate references, 357 articles were considered for the pooled individual data analysis. 297 studies were excluded after screening the title and abstract because they were review articles, editorials, opinion papers and guidelines. A total of 47 studies with individual-level data on our variables of interest qualified for inclusion, identifying
98 reported patients with COVID-19 and ICH. A further 13 studies reported only aggregate-level data on patients with COVID-19-associated intracranial haemorrhage not already included in our individual patient data meta-analysis. These contained 190 cases of intracranial haemorrhage, of which 99 were intracerebral. Five studies including 41 patients with ICH reported ICH-specific data (see Table 1) and were eligible for our two-stage pooled analysis. 
Table 1 Aggregate data summary

\begin{tabular}{llll}
\hline Paper & Setting & $\begin{array}{l}\text { Number of } \\
\text { ICH cases }\end{array}$ & Data available \\
\hline Trifan 2020 [6] & Multihospital case series, US & 16 & Sex, ICH topography, anticoagulation \\
Dogra 2020 [20] & Multihospital case series, US & 5 & Age, sex, risk factors, anticoagulation \\
Nawabi 2020 [37] & Multihospital case series, Europe & 6 & ICH topography \\
Rothstein 2020 [38] & $\begin{array}{c}\text { Multihospital case series, US; } \\
\text { three patients on ECMO }\end{array}$ & 5 & ICH topography, anticoagulation \\
Altschul 2020 [39] & Multihospital case series, US & 9 & Age, sex, risk factors, anticoagulation \\
\hline
\end{tabular}

\section{Pooled individual patient data analysis}

\section{Patient demographics and intracerebral haemorrhage characteristics}

The characteristics of the patients included in our pooled individual patient data analysis $(n=98)$ are shown in Table 2; 79\% (95\% CI 70.0-86.9\%) had critically severe COVID-19. Detailed data from all individual patients included are shown in Supplementary Table 2. The median age was 60 years (IQR 53-67); 64\% were men. The proportions of patients with vascular risk factors were: hypertension in $53 \%$, diabetes in $29 \%$, dyslipidaemia in $16 \%$, high BMI in $11 \%$ and history of smoking in 11\% (Table 2); $20 \%$ had no history of vascular risk factors.

ICH was frequently lobar (67\%), multifocal (36\%) and severe, with a median NIHSS score of 28 (IQR 15-28; available in 18 patients). Non-lobar (deep or infratentorial) ICHs were less common (29\%), while mixed (lobar and nonlobar) ICHs were reported in only $4 \%$ of cases. Coagulopathy was the most common reported cause of ICH (50/98; $51 \%)$, nearly always attributed to therapeutic anticoagulation (48/50; 96\%).

$65 / 91(71 \%)$ patients were receiving anticoagulation at the time of ICH, which was therapeutic in 53/91 (58\%): therapeutic unfractionated heparin (UFH) in $36(40 \%)$; therapeutic low molecular weight heparin (LMWH) in 14 (15\%); and warfarin in $3(3 \%)$. Prophylactic LMWH was used in 12/91 (13\%). The indication for anticoagulation was extracorporeal membrane oxygenation (ECMO) in 20, concurrent thromboembolic events (pulmonary embolism and deep venous thrombosis) in 5, atrial fibrillation in 3, mechanical cardiac valve replacement in one patient and occlusive femoral artery thrombus in one. 35 patients were started on either therapeutic or prophylactic anticoagulation for suspected COVID-19 hypercoagulability based on local protocols.

The characteristics of patients with known anticoagulation status are shown in Table 3. 26/91 (29\%, 95\% CI 20-39\%) patients were not receiving any anticoagulation at the time of ICH. Of these, $12 / 26(46 \%, 95 \%$ CI $27-67 \%)$ were lobar, $2 / 23(9 \%, 95 \%$ CI 1-28\%) were multifocal,
$7 / 26(27 \%, 95 \%$ CI $12-48 \%)$ were attributed to hypertensive arteriopathy, and $1 / 26(4 \%, 95 \%$ CI $0-20 \%)$ was associated with coagulopathy. The proportions of patients with lobar $\mathrm{ICH}$ and multifocal ICH were lower in those who were not anticoagulated compared to those who were anticoagulated $(12 / 26(46 \%, 27-67 \%)$ vs $51 / 63(81 \%, 69-90 \%), p=0.002$; and $(2 / 23(9 \%, 1-28 \%)$ vs $31 / 63(49 \%, 36-62 \%), p<0.001$, respectively). The proportion of ICH attributed to coagulopathy was $1 / 26(4 \%, 0-20 \%)$ in patients who were not anticoagulated compared to $49 / 65(75 \%, 63-85 \%)$ in those who were $(\mathrm{p}<0.001)$. The proportion of ICH attributed to hypertensive arteriopathy was higher in patients who were not anticoagulated compared to those who were (7/26 (27\%, $12-48 \%)$ vs $0 / 65(0 \%, 0-5 \%), p<0.001)$.

Coagulability markers are summarized in Table 2. D-dimers were reported in 64/98 (65\%) patients; they were elevated $(>500 \mu \mathrm{g} / \mathrm{L})$ in $94 \%$ of cases with a median of $3387 \mu \mathrm{g} / \mathrm{L}$ (IQR 1745-5670).

\section{Coronavirus disease severity}

The time of onset of COVID-19 symptoms (where present) in relation to ICH was reported for 92/98 (94\%) patients. Of these, 71 (77\%) developed symptoms before ICH with a median interval of 15 days (IQR 5-20), while 21 (23\%) had no symptoms at the time of ICH. 77 (79\%) were critically ill (developed respiratory failure requiring mechanical ventilation or septic shock or other organ dysfunction or failure that required intensive care [11]) including 20 (21\%) who received extracorporeal membrane oxygenation (ECMO) treatment. A further four patients (4\%) had severe disease (with one of the following criteria: tachypnoea $\geq 30$ breaths per min; or $\mathrm{O} 2$ sat $\leq 93 \%$ at rest; or $\mathrm{PaO} 2 /$ $\mathrm{FiO} 2$ ratio $<300 \mathrm{mmHg}$ [11]), 9 (9\%) had moderate disease activity (with fever, respiratory tract symptoms and pneumonia on imaging [11]), 2 (2\%) had mild symptoms and no pneumonia on imaging and $5(5 \%)$ were asymptomatic. 
Table 2 Characteristics of patients included in individual patient data pooled analysis

\begin{tabular}{|c|c|}
\hline \multicolumn{2}{|l|}{ Demographics $(n=98)$} \\
\hline Age - Median (IQR) & $60(53-67)$ \\
\hline Male \% $(95 \% \mathrm{CI})$ & $64 \%(54-73.7 \%)$ \\
\hline Vascular risk factors: $(n=91)$ & $N(\%, 95 \% \mathrm{CI})$ \\
\hline Hypertension & $48(53 \%, 42.0-63.3 \%)$ \\
\hline Diabetes & $26(29 \%, 19.6-39.0 \%)$ \\
\hline Dyslipidaemia & $15(16 \%, 9.5-25.7 \%)$ \\
\hline Smoking & $10(11 \%, 5.4-19.3 \%)$ \\
\hline High body mass index & $10(11 \%, 5.4-19.3 \%)$ \\
\hline Atrial fibrillation & $3(3 \%, 0.7-9.3 \%)$ \\
\hline Ischemic heart disease & $3(3 \%, 0.7-9.3 \%)$ \\
\hline Alcohol & $2(2 \%, 0.3-7.7 \%)$ \\
\hline No previous medical history & $18(20 \%, 12.1-29.5 \%)$ \\
\hline Intracerebral haemorrhage characteristics & $N(\%, 95 \% \mathrm{CI})$ \\
\hline \multicolumn{2}{|l|}{ Location $(n=96)$} \\
\hline Lobar & $64(67 \%, 56.3-76.0 \%)$ \\
\hline Non-lobar (deep or infratentorial) & $28(29 \%, 20.3-39.3 \%)$ \\
\hline Mixed (lobar and non-lobar) & $4(4 \%, 1.15-10.3 \%)$ \\
\hline \multicolumn{2}{|l|}{ Multifocality $(n=91)$} \\
\hline Multifocal & $33(36 \%, 26.4-47.0 \%)$ \\
\hline Focal & $58(64 \%, 53.0-73.6 \%)$ \\
\hline \multicolumn{2}{|l|}{ Aetiology $(n=98)$} \\
\hline Coagulopathy & $50(51 \%, 40.7-61.3 \%)$ \\
\hline Hypertensive arteriopathy & $11(11 \%, 5.7-19.2 \%)$ \\
\hline Cerebral amyloid angiopathy & $1(1 \%, 0.0-5.6 \%)$ \\
\hline Macrovascular causes $(*)$ & $3(3 \%, 0.6-8.7 \%)$ \\
\hline Cerebral venous thrombosis & $1(1 \%, 0.0-5.6 \%)$ \\
\hline Moya-Moya disease & $1(1 \%, 0.0-5.6 \%)$ \\
\hline Undetermined & $31(32 \%, 22.6-41.8 \%)$ \\
\hline Anticoagulation $(n=91)$ & $N(\%, 95 \% \mathrm{CI})$ \\
\hline Therapeutic unfractionated heparin & $36(40 \%, 29.5-50.4 \%)$ \\
\hline Therapeutic low molecular weight heparin & $14(15 \%, 8.7-24.5 \%)$ \\
\hline Prophylactic low molecular weight heparin & $12(13 \%, 7.0-21.9 \%)$ \\
\hline Warfarin & $3(3 \%, 0.7-9.3 \%)$ \\
\hline No anticoagulation & $26(29 \%, 19.6-39.0 \%)$ \\
\hline COVID-19 & $N(\%, 95 \% \mathrm{CI})$ \\
\hline \multicolumn{2}{|l|}{ Prior symptoms $(n=92)$} \\
\hline Prior COVID-19 symptoms & $71(77 \%, 67.3-85.3 \%)$ \\
\hline No prior COVID-19 symptoms & $21(23 \%, 14.7-32.8 \%)$ \\
\hline \multicolumn{2}{|l|}{ Days since COVID-19 symptoms $(n=68)$} \\
\hline Median, IQR & $15(5-20)$ \\
\hline \multicolumn{2}{|l|}{ Severity $(n=97)$} \\
\hline Critical & $77(79 \%, 70.0-86.9 \%)$ \\
\hline Severe & $4(4 \%, 1.1-10.2 \%)$ \\
\hline Moderate & $9(9 \%, 4.3-16.9 \%)$ \\
\hline Mild & $2(2 \%, 0.3-7.3 \%)$ \\
\hline Asymptomatic & $5(5 \%, 1.7-11.6 \%)$ \\
\hline $\operatorname{ECMO}(n=98)$ & $20(21 \%, 12.9-29.8 \%)$ \\
\hline \multicolumn{2}{|l|}{ Outcome $(n=98)$} \\
\hline Deceased & $53(54 \%, 43.7-64.2 \%)$ \\
\hline
\end{tabular}

Table 2 (continued)

\begin{tabular}{cl}
\hline Critically ill & $18(18 \%, 11.3-27.5 \%)$ \\
Discharged to rehabilitation unit or home & $27(28 \%, 19.0-37.5 \%)$ \\
Coagulability markers & Median, IQR \\
D-Dimer $(\mu \mathrm{g} / \mathrm{L})(n=64)$ & $3387(1745-5670)$ \\
Fibrinogen $(\mathrm{g} / \mathrm{L}):(n=42)$ & $5(3.6-6.5)$ \\
aPTT $(\mathrm{s})(n=36)$ & $32(25-59.5)$ \\
PTT $(\mathrm{s})(n=21)$ & $64(41.6-89.3)$ \\
INR $(n=45)$ & $1.2(1.1-1.5)$ \\
Prothrombin time $(\mathrm{s})(n=28)$ & $12.8(11.7-15.7)$ \\
Platelet count $(/ \mathrm{mm} 3)(n=72)$ & $211(143,288.5 \mathrm{k})$ \\
CRP $(\mathrm{mg} / \mathrm{L})(n=57)$ & $74(13-130)$ \\
Serum ferritin $(\mu \mathrm{g} / \mathrm{L})(n=20)$ & $1554(983-2289)$ \\
\hline
\end{tabular}

* Macrovascular causes (aneurysm, arteriovenous malformation, cavernous malformation)

ECMO extracorporeal membrane oxygenation, $A P T T$ activated partial thromboplastin time, $P T T$ prothrombin time, INR international normalised ratio, $C R P$ C-reactive protein

\section{Outcome}

The mortality was 53/98 (54\%); 18/98 (18\%) remained critically ill and 27/98 (28\%) were discharged home or to a rehabilitation unit.

\section{Pooled analysis of individual patient and aggregate data}

Our two-stage pooled analysis included the pooled individual patient data (Table $2, n=98$ ) and ICH-specific aggregate-level data (Table 1, $n=41$ ). The results for key variables are shown in Fig. 2. The mean age was 57.9 years (95\% CI 55.5-60.4 years). The estimated proportion of patients of the male sex was $73.9 \%$ (95\% CI 66.0-81.2\%). 53.6\% (95\% CI 43.1-63.9\%) were hypertensive, and $74.4 \%$ (95\% CI 46.6-95.4\%) were prescribed therapeutic-dose anticoagulation at the time of ICH. 67.7\% (95\% CI 46.6-86.0) of ICH were lobar, and $20.1 \%$ (95\% CI 4.7-40.6\%) were multifocal.

\section{Incidence of ICH in COVID-19}

Twelve cohort studies ( $n=63,390$ patients) reported data on the incidence of ICH in COVID-19 inpatients, with the incidence ranging from 0.13 to $2.03 \%$. The pooled incidence of ICH across these studies was $0.38 \%$ (95\% CI $0.22-0.58 \%$ ) (Fig. 3). 
Table 3 ICH characteristics in non-anticoagulated versus anticoagulated patients

\begin{tabular}{lll}
\hline & Non-anticoagulated $(\%, 95 \%$ CI) & Anticoagulated (\%, 95\% CI) \\
\hline Patients & $26 / 91(29 \%, 20-39 \%)$ & $65 / 91(71 \%, 61-80 \%)$ \\
Location & Available in 26/26 & Available in 63/65 \\
Lobar & $12 / 26(46 \%, 27-67 \%)$ & $51 / 63(81 \%, 69-90 \%)$ \\
Non-lobar (deep or infratentorial) & $14 / 26(54 \%, 33-73 \%)$ & $8 / 63(13 \%, 6-24 \%)$ \\
Mixed (lobar and non-lobar) & $0 / 26(0 \%, 0-11 \%)$ & $4 / 63(6 \%, 2-15 \%)$ \\
Focality & Available in 23/26 & Available in 63/65 \\
Multifocal & $2 / 23(9 \%, 1-28 \%)$ & $31 / 63(49 \%, 36-62 \%)$ \\
Focal & $21 / 23(91 \%, 72-99 \%)$ & $32 / 63(51 \%, 38-64 \%)$ \\
Mechanism & Available in 26/26 & Available in 65/65 \\
Coagulopathy & $1 / 26(4 \%, 0-20 \%)$ & $49 / 65(75 \%, 63-85 \%)$ \\
Hypertensive arteriopathy & $7 / 26(27 \%, 12-48 \%)$ & $0 / 65(0 \%, 0-5 \%)$ \\
CAA & $1 / 26(4 \%, 0-20 \%)$ & $0 / 65(0 \%, 0-5 \%)$ \\
CVT & $0 / 26(0 \%, 0-11 \%)$ & $1 / 65(2 \%, 0-8 \%)$ \\
Moya-Moya disease & $1 / 26(4 \%, 0-20 \%)$ & $0 / 65(0 \%, 0-5 \%)$ \\
Macrovascular causes & $2 / 26(8 \%, 1-25 \%)$ & $1 / 65(2 \%, 0-8 \%)$ \\
Undetermined & $14 / 26(54 \%, 33-73 \%)$ & $14 / 65(22 \%, 12-33 \%)$ \\
\hline
\end{tabular}

\section{Discussion}

Spontaneous (non-traumatic) ICH in patients with COVID19 appears to have distinct characteristics that differ from those previously reported for ICH not associated with COVID-19. Based on our pooled individual patient data analysis, in comparison to non-COVID-19 populations $[12,13]$, ICH associated with COVID-19 seems to affect a younger (median age 60 ), more frequently male $(64 \%)$ and less often hypertensive (53\%) population, and appears to be more often lobar, multifocal, and associated with the use of anticoagulant drugs. Our pooled analysis of individual and aggregate data showed similar findings. These results are potentially relevant for understanding underlying mechanisms, and for the management of patients with COVID19 , including the use of anticoagulants to treat or prevent thromboembolic complications. We found a low incidence of ICH in patients with COVID-19 (0.38\%), but the high mortality rate $(54 \%)$ suggests that ICH is among the most severe neurological complications of COVID-19. Furthermore, our data suggest that the majority of ICH occur in the context of critically severe COVID-19.

The high prevalence of lobar ICH (in 67\% of patients in our pooled individual patient analysis, and $67.7 \%$ in the pooled analysis of individual and aggregate data) is striking, and consistent with the pattern recently described in a systematic review of neuroimaging features of COVID-19 [14]. In non-COVID-19 ICH populations, lobar ICH typically accounts for only $32-38 \%$ of cases [15-17]. In nonCOVID-19 cohorts, lobar ICHs are mainly due to either macrovascular causes (arteriovenous malformations or aneurysms), hyaline arteriolosclerosis or cerebral amyloid angiopathy [18].
Another important finding was that ICH in COVID-19 was multifocal in $36 \%$ of patients in our pooled individual patient analysis and $20.6 \%$ in our two-stage pooled analysis; this is much more frequent than in typical ICH cohorts in which ICH are multiple in only about $6 \%$ of cases [19]. Interestingly this previous study also found that multiple ICHs were more often lobar and associated with systemic coagulopathy [19]. We found hypertension in only $53 \%$ of cases, also in keeping with the data in multiple non-COVID-19 ICH [19]. In COVID-19 patients not on anticoagulants, the proportion of multifocal ICH was much lower (9\%) than in our full cohort (49\%), and similar to the general rate of ICH multifocality in non-COVID-19 ICH populations, suggesting that anticoagulation is an important precipitating factor for multifocal ICH in patients with COVID-19.

The unusual characteristics of ICH associated with COVID-19 raise the possibility that the underlying mechanisms might be different from those of spontaneous ICH in patients without COVID-19. In our pooled individual and aggregate data analysis, $74.4 \%$ of ICH were associated with therapeutic anticoagulation, which may have been an important contributory factor. It is also possible that ICH occurs in more severe COVID-19, in which anticoagulation is more likely to be given (including for ECMO). Our findings are consistent with a recent study reporting 33 patients with COVID-19 and intracranial bleeding (primary ICH in 5 patients and haemorrhagic transformation of an infarct in most of the rest) and a high rate of anticoagulation $(66.7 \%$ therapeutic anticoagulation and $9.1 \%$ prophylactic anticoagulation) [20]. In another study, therapeutic anticoagulation use (compared with not using anticoagulation) in COVID-19 patients was associated with a five-fold increased risk of ICH [21]. By contrast, in previous mechanistic classifications of 


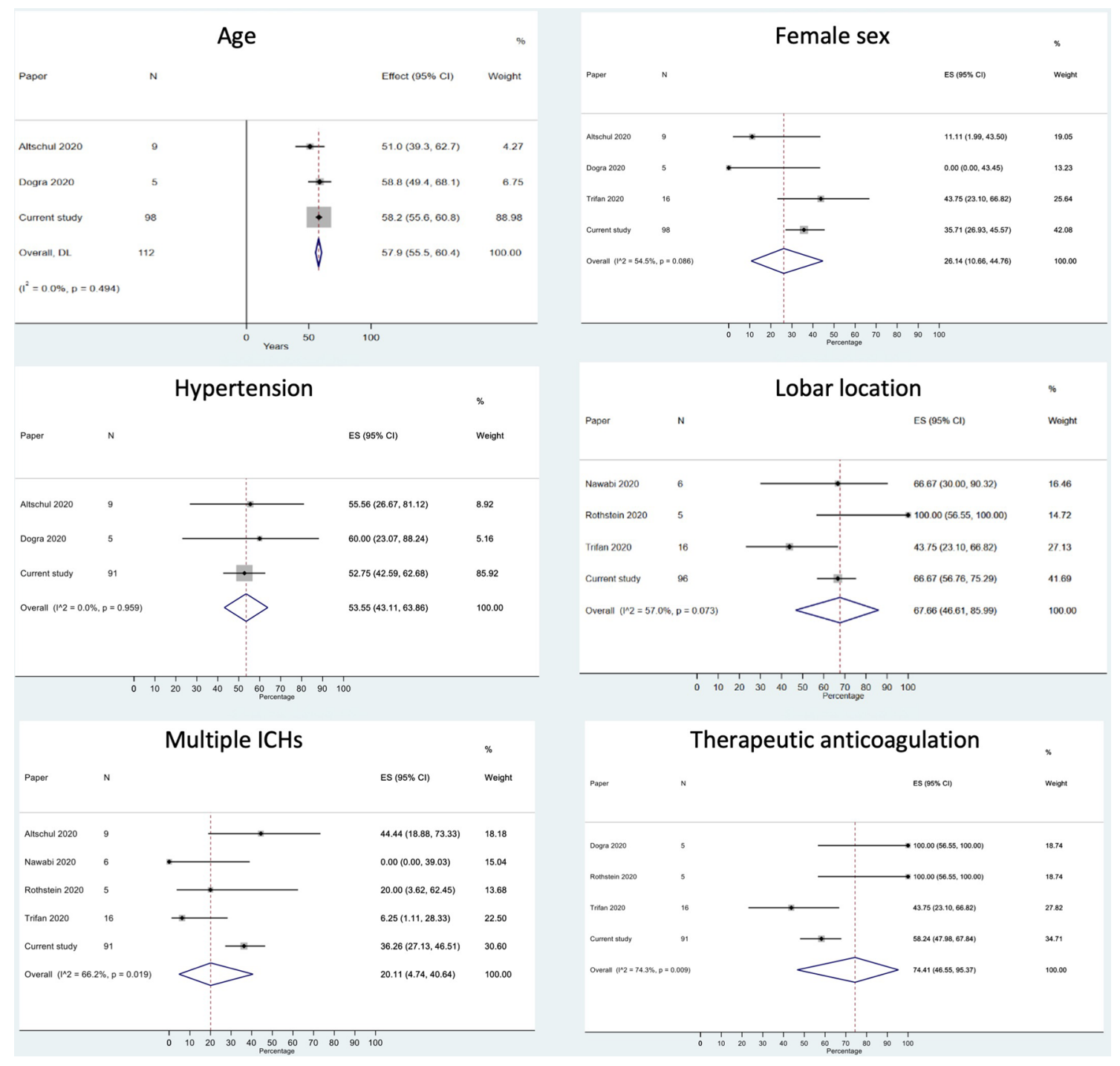

Fig. 2 ICH characteristics in our two-stage pooled analysis of individual patient and aggregate data

non-COVID-19 ICH, anticoagulation accounted for only $10-15 \%$ of cases [22]. Interestingly, only $4 \%$ of ICHs were associated with coagulopathy in non-anticoagulated patients, supporting the hypothesis that COVID-19-associated ICH can result from vessel wall pathology rather than requiring the presence of impaired clotting associated with a critical illness, anticoagulation therapy, or both.

D-dimer levels were high in most patients (median (IQR) $3387 \mu \mathrm{g} / \mathrm{L}$ (IQR 1745-5670), surpassing the threshold identified as a predictor of in-hospital mortality [23] and consistent with a recent study in COVID-19 associated acute stroke that found elevated D-Dimers in all patients with haemorrhagic stroke (mean of $3,387 \mu \mathrm{g} / \mathrm{L}$ ), which was lower than the levels observed in ischaemic stroke (mean $7148 \mu \mathrm{g} / \mathrm{L}$ ) [24]. Arterial and venous thromboembolic events, including ischaemic strokes, have been widely associated with severe coronavirus disease [25], while cerebrovascular disease is associated with increased disease severity in patients with COVID-19 [26]. Similarly, we found that the majority of ICH $(79 \%)$ occurred in patients with critically severe COVID-19. These findings, together with the findings from the current study, emphasise the challenging combination of thrombotic and haemorrhagic complications, particularly in severe COVID-19 [27].

The pathogenesis of intracerebral haemorrhage in COVID-19 patients is likely to be complex. One report describing 41 cases of COVID-19 indicated that prolonged prothrombin time, elevated D-dimer, and severe platelet reduction occur in critical COVID-19 patients [28], all of which are associated with increased propensity for haemorrhagic complications. Although anticoagulation was common in our study, it is not considered to be a sufficient or 


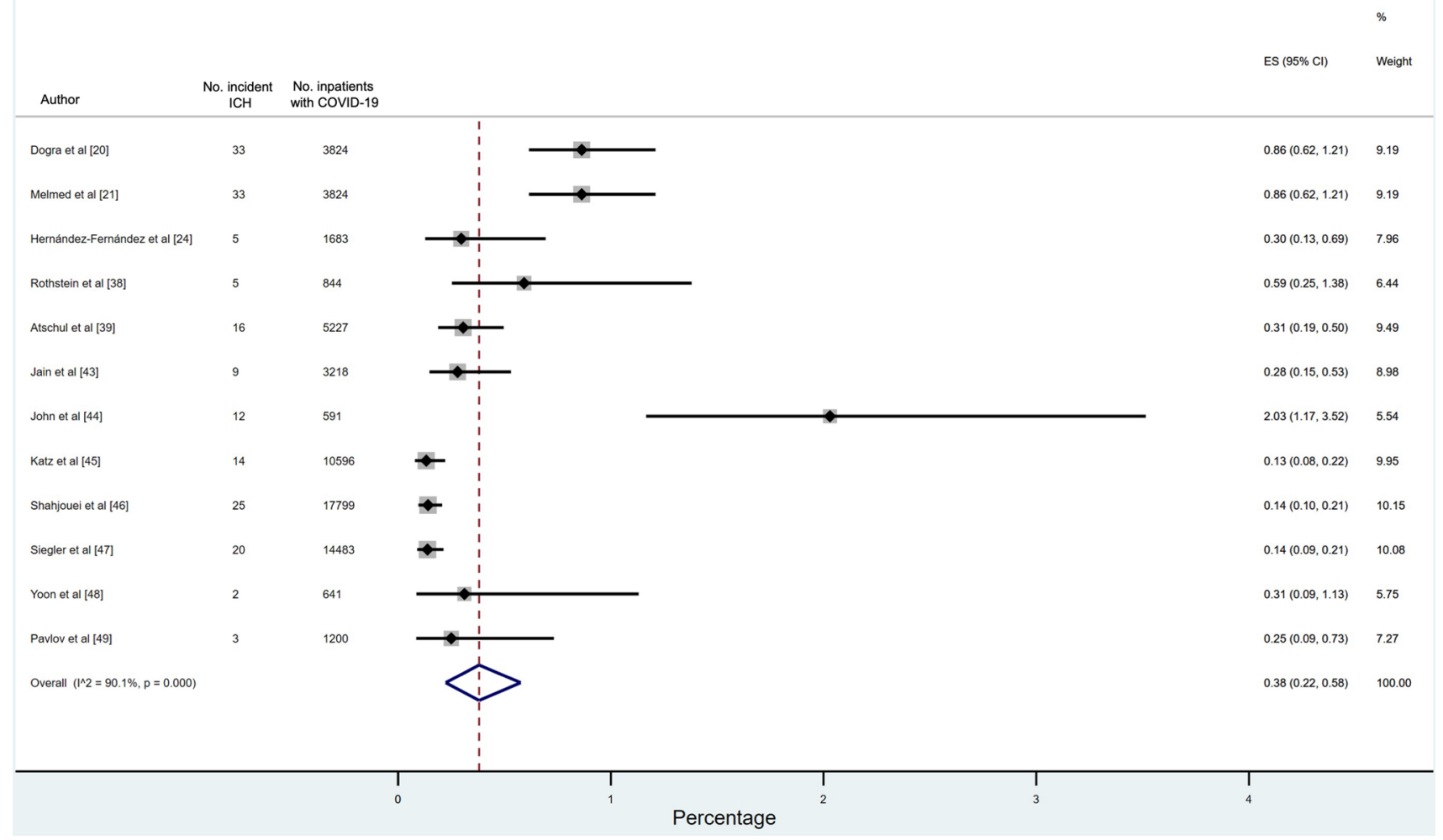

Fig. 3 Incidence of ICH in inpatients with COVID-19

necessary cause for ICH but is hypothesised to aggravate haemorrhage from vessels prone to bleeding due to other pathologies. SARS-CoV-2 has both direct and indirect effects on the cerebral vasculature that could increase the risk of haemorrhage through various potential mechanisms [29]. First, it has been widely reported that SARS-CoV-2 binds to the angiotensin-converting enzyme 2 (ACE2) receptors largely present in the brain vessels [30] which could cause endothelial injury and blood-brain barrier (BBB) disruption [31]. Endothelial cell infection and endotheliitis have been demonstrated in COVID-19 via histological identification of viral elements within endothelial cells and an accumulation of inflammatory cells and apoptosis [32]. A diffuse bleeding-prone endotheliitis, perhaps aggravated by the use of therapeutic anticoagulation, could thus be a contributory factor in explaining our findings. Other studies in COVID-19 have found evidence of a haemorrhagic leukoencephalopathy with multiple cerebral microbleeds, in keeping with the presence of a diffuse haemorrhage-prone vasculopathy [33]. Other accumulating evidence suggests that SARS-CoV-2 may be associated with a cytokine storm syndrome and excessive oxidative stress [34], which could disrupt endothelial function, causing blood-brain-barrier breakdown and ICH [35]. Finally, disruption of the renin-angiotensin system (RAS) may also play a role in COVID-19-mediated ICH; down-regulation of endothelial ACE2 receptors can cause dys-autoregulation of cerebral blood flow [36].

The majority of patients with COVID-19-associated ICH (77\%) presented with COVID-19 symptoms prior to haemorrhagic stroke onset, with a median delay of 15 days in keeping with previous studies $[37,38]$, also suggesting that a delayed haemorrhagic vasculopathy could be a contributory factor.

In our review, $79 \%$ of reported patients were critically ill requiring ICU admission or mechanical ventilation, with very high mortality (54\%), consistent with previous studies reporting that ICH was more likely in patients with severe pulmonary COVID-19 (81.8\%) with an extremely poor overall prognosis and a mortality rate of $63.6 \%$ [39]. Furthermore, $21 \%$ of patients received ECMO in our study, which may increase the risk of thrombotic and bleeding complications as indicated by a recent case series that reported 4 out of 10 patients with COVID-19 placed on ECMO developed intracranial haemorrhage, 3 of whom died [40]. By contrast, in non-COVID-19 cohorts, the incidence of ECMO-associated ICH varied between 1.8 and 21\% [41].

The incidence of ICH in COVID-19 patients in our study was $0.38 \%$ which is lower than the reported incidence of acute ischemic stroke in COVID-19 patients which ranges from 0.9 to $2.7 \%$ [42]. 
The main strength of our study is the inclusion of all available individual patient and aggregate data regarding ICH in patients with COVID-19, allowing us to describe distinct characteristics that are not clearly apparent in small case series. In addition, our review highlights that COVID19 can be associated with both prothrombotic and haemorrhage-prone states, which might be relevant for anticoagulation strategies.

We acknowledge several limitations of our study. First, we included hospital case series which are likely to be affected by selection and publication bias. Second, we did not have access to data from a contemporaneous control group of patients with non-COVID-19 associated ICH; comparison to historical ICH controls is not likely to be helpful because of the change in the spectrum of stroke presenting to hospital associated with the COVID-19 pandemic [50]. Third, we did not have access to detailed investigations including neuroimaging markers of small vessel disease or vascular imaging to exclude macrovascular causes and assign causes in a systematic and standardised way across cohorts; moreover, limited MRI access during the pandemic might have led to misclassification of haemorrhagic transformation of infarcts as ICHs. Finally, due to the limitations in published data, we were not able to report on the causes of death (for example the proportions attributed to respiratory or neurological disease).

Nevertheless, despite limitations related to the small patient numbers described in most studies, our findings suggest that ICH in COVID-19 has distinct characteristics with potential implications for understanding mechanisms, management of anticoagulation, and clinical trials. Ongoing randomised controlled trials to clarify the risk, benefit, and optimal dose of anticoagulation in patients with COVID-19 should include ICH as a key safety outcome.

Supplementary Information The online version contains supplementary material available at https://doi.org/10.1007/s00415-021-10425-9.

\section{Compliance with ethical standards}

Conflict of interest On behalf of all authors, the corresponding author states that there is no conflict of interest.

Open Access This article is licensed under a Creative Commons Attribution 4.0 International License, which permits use, sharing, adaptation, distribution and reproduction in any medium or format, as long as you give appropriate credit to the original author(s) and the source, provide a link to the Creative Commons licence, and indicate if changes were made. The images or other third party material in this article are included in the article's Creative Commons licence, unless indicated otherwise in a credit line to the material. If material is not included in the article's Creative Commons licence and your intended use is not permitted by statutory regulation or exceeds the permitted use, you will need to obtain permission directly from the copyright holder. To view a copy of this licence, visit http://creativecommons.org/licenses/by/4.0/.

\section{References}

1. Tang N, Li D, Wang X, Sun Z (2020) Abnormal coagulation parameters are associated with poor prognosis in patients with novel coronavirus pneumonia. J Thromb Haemost 18(4):844-847

2. Han H, Yang L, Liu R, Liu F, Wu KL, Li J et al (2020) Prominent changes in blood coagulation of patients with SARSCoV-2 infection. Clin Chem Lab Med. https://doi.org/10.1515/ cclm-2020-0188

3. Beyrouti R, Adams ME, Benjamin L et al (2020) Characteristics of ischaemic stroke associated with COVID-19. J Neurol Neurosurg Psychiatry 91:889-891

4. Moores LK, Tritschler T, Brosnahan S et al (2020) Prevention, diagnosis, and treatment of vte in patients with coronavirus disease 2019: CHEST guideline and expert panel report. Chest 158(3):1143-1163. https://doi.org/10.1016/j.chest.2020.05.559

5. Tang N, Bai H, Chen X, Gong J, Li D, Sun Z (2020) Anticoagulant treatment is associated with decreased mortality in severe coronavirus disease 2019 patients with coagulopathy. J Thromb Haemost. https://doi.org/10.1111/jth.14817

6. Trifan G, Goldenberg FD, Caprio FZ et al (2020) Characteristics of a diverse cohort of stroke patients with SARS-CoV-2 and outcome by sex. J Stroke Cerebrovasc Dis 29(11):105314. https://doi. org/10.1016/j.jstrokecerebrovasdis.2020.105314

7. Ellul MA, Benjamin L, Singh B et al (2020) Neurological associations of COVID-19. Lancet Neurol 19(9):767-783. https://doi. org/10.1016/S1474-4422(20)30221-0

8. Moher D, Shamseer L, Clarke M et al (2015) Preferred reporting items for systematic review and meta-analysis protocols (PRISMA-P) 2015 statement. Syst Rev 4(1):1

9. WHO Clinical management of severe acute respiratory infection when novel coronavirus (nCoV) infection is suspected 2020. https ://www.who.int/publications-detail/clinical-management-of-sever e-acute-respiratory-infection-when-novel-coronavirus-(ncov)infection-is-suspected. Accessed 27 Oct 2020

10. Riley RD, Simmonds MC, Look MP (2007) Evidence synthesis combining individual patient data and aggregate data: a systematic review identified current practice and possible methods. J Clin Epidemiol 60(5):431-439. https://doi.org/10.1016/j.jclin epi.2006.09.009 (PMID: 17419953)

11. Chinese clinical guidance for COVID-19 pneumonia diagnosis and treatment (7th edition) https://www.acc.org/latest-in-cardi ology/articles/2020/03/17/11/22/chinese-clinical-guidance-forcovid-19-pneumonia-diagnosis-and-treatment. Accessed 27 Oct 2020

12. An SJ, Kim TJ, Yoon BW (2017) Epidemiology, risk factors, and clinical features of intracerebral hemorrhage: an update. J Stroke 19:3-10. https://doi.org/10.5853/jos.2016.00864

13. Javalkar V, Kuybu O, Davis D, Kelley RE (2020) Factors associated with inpatient mortality after intracerebral hemorrhage: updated information from the United States nationwide inpatient sample. J Stroke Cerebrovasc Dis 29:104583. https://doi. org/10.1016/j.jstrokecerebrovasdis.2019.104583

14. Ladopoulos T, Zand R, Shahjouei S, Chang JJ, Motte J, Charles James J, Katsanos AH, Kerro A, Farahmand G, Vaghefi Far A, Rahimian N, Ebrahimzadeh SA, Abedi V, Papathanasiou M, Labedi A, Schneider R, Lukas C, Tsiodras S, Tsivgoulis G, Krogias C (2021) COVID-19: neuroimaging features of a pandemic. J Neuroimaging. https://doi.org/10.1111/jon.12819 (PMID: 33421032)

15. Safatli DA, Günther A, Schlattmann P, Schwarz F, Kalff R, Ewald $\mathrm{C}$ (2016) Predictors of 30-day mortality in patients with spontaneous primary intracerebral hemorrhage. Surg Neurol Int 7(Suppl 18):S510-S517. https://doi.org/10.4103/2152-7806.187493 (PMID: 27583176; PMCID: PMC4982350) 
16. Rannikmäe K, Woodfield R, Anderson CS et al (2016) Reliability of intracerebral hemorrhage classification systems: a systematic review. Int J Stroke 11(6):626-636. https://doi.org/10.1177/17474 93016641962

17. Seiffge DJ, Curtze S, Dequatre-Ponchelle N, Pezzini A, Tatlisumak T, Cordonnier C, Werring D (2019) Hematoma location and morphology of anticoagulation-associated intracerebral hemorrhage. Neurology 92(8):e782-e791. https://doi.org/10.1212/ WNL.0000000000006958 (PMID: 30674603)

18. Wilson D, Charidimou A, Werring DJ (2014) Advances in understanding spontaneous intracerebral hemorrhage: insights from neuroimaging. Expert Rev Neurother 14(6):661-678

19. Wu TY, Yassi N, Shah DG, Ma M, Sharma G, Putaala J et al (2017) Simultaneous multiple intracerebral hemorrhages (SMICH). Stroke 48(3):581-586. https://doi.org/10.1161/strok eaha.116.015186

20. Dogra S, Jain R, Cao M et al (2020) Hemorrhagic stroke and anticoagulation in COVID-19. J Stroke Cerebrovasc Dis 29(8):104984. https://doi.org/10.1016/j.jstrokecerebrovasdis .2020 .104984

21. Melmed KR, Cao M, Dogra S et al (2020) Risk factors for intracerebral hemorrhage in patients with COVID-19. J Thromb Thrombolysis. https://doi.org/10.1007/s11239-020-02288-0

22. Flaherty ML, Kissela B, Woo D, Kleindorfer D, Alwell K, Sekar P, Moomaw CJ, Haverbusch M, Broderick JP (2007) The increasing incidence of anticoagulant-associated intracerebral hemorrhage. Neurology 68(2):116-121. https://doi.org/10.1212/01. wnl.0000250340.05202.8b (PMID: 17210891)

23. Zhang L, Yan X, Fan Q, Liu H, Liu X, Liu Z et al (2020) D-dimer levels on admission to predict in-hospital mortality in patients with Covid-19. J Thromb Haemost. https://doi.org/10.1111/ jth.14859

24. Hernández-Fernández F, Valencia HS, Barbella-Aponte RA et al (2020) Cerebrovascular disease in patients with COVID-19: neuroimaging, histological and clinical description. Brain. https://doi. org/10.1093/brain/awaa239

25. Klok FA, Kruip MJHA, van der Meer NJM, Arbous MS, Gommers D, Kant KM, Kaptein FHJ, van Paassen J, Stals MAM, Huisman MV, Endeman H (2020) Confirmation of the high cumulative incidence of thrombotic complications in critically ill ICU patients with COVID-19: an updated analysis. Thromb Res 191:148-150. https://doi.org/10.1016/j.thromres.2020.04.041

26. Aggarwal G, Lippi G, Michael Henry B (2020) Cerebrovascular disease is associated with an increased disease severity in patients with coronavirus disease 2019 (COVID-19): a pooled analysis of published literature. Int J Stroke 15:385-389

27. Connors JM, Levy JH (2020) Covid-19 and its implications for thrombosis and anticoagulation. Blood 135:2033

28. Huang C, Wang Y, Li X, Ren L, Zhao J, Hu Y, Zhang L, Fan G, Xu J, Gu X, Cheng Z, Yu T, Xia J et al (2020) Clinical features of patients infected with 2019 novel coronavirus in Wuhan, China. Lancet 395:497-506. https://doi.org/10.1016/S0140 -6736(20)30183-5 (PMID: 31986264)

29. Dong S, Liu P, Luo Y, Cui Y, Song L, Chen Y (2020) Pathophysiology of SARS-CoV-2 infection in patients with intracerebral hemorrhage. Aging 12(13):13791-13802. https://doi. org/10.18632/aging.103511

30. Xia H, Lazartigues E (2008) Angiotensin-converting enzyme 2 in the brain: properties and future directions. J Neurochem 107(6):1482-1494

31. Hoffmann M, Kleine-Weber H, Schroeder S et al (2020) SARSCoV-2 entry depends on ACE2 and TMPRSS2 and is blocked by a clinically proven protease inhibitor. Cell 181(271-80):e8

32. Varga Z, Flammer AJ, Steiger P, Haberecker M, Andermatt R, Zinkernagel AS et al (2020) Endothelial cell infection and endotheliitis in COVID-19. Lancet 395(10234):1417-1418
33. Radmanesh A, Derman A, Lui YW et al (2020) COVID-19-associated diffuse leukoencephalopathy and microhemorrhages. Radiology 297(1):E223-E227. https://doi.org/10.1148/radiol.20202 02040

34. Chen G, Wu D, Guo W, Cao Y, Huang D, Wang H et al (2020) Clinical and immunological features of severe and moderate coronavirus disease 2019. J Clin Invest 130:2620-2629. https://doi. org/10.1172/JCI137244

35. Wilkinson DA, Pandey AS, Thompson BG, Keep RF, Hua Y, Xi $G$ (2018) Injury mechanisms in acute intracerebral hemorrhage. Neuropharmacology 134:240-248

36. Zhang H, Penninger JM, Li Y, Zhong N, Slutsky AS (2020) Angiotensin-converting enzyme 2 (ACE2) as a SARS-CoV-2 receptor: molecular mechanisms and potential therapeutic target. Intensive Care Med 46(4):586-590

37. Nawabi J, Morotti A, Wildgruber M et al (2020) Clinical and imaging characteristics in patients with SARS-CoV-2 infection and acute intracranial hemorrhage. J Clin Med 9(8):2543. https:// doi.org/10.3390/jcm9082543

38. Rothstein A, Oldridge O, Schwennesen H, Do D, Cucchiara BL (2020) Acute cerebrovascular events in hospitalized COVID-19 patients. Stroke 51(9):e219-e222. https://doi.org/10.1161/STROK EAHA.120.030995 (PMID: 32684145; PMCID: PMC7386677)

39. Altschul DJ, Unda SR, de La Garza RR, Zampolin R, Benton J, Holland R, Fortunel A, Haranhalli N (2020) Hemorrhagic presentations of COVID-19: risk factors for mortality. Clin Neurol Neurosurg 198:106112. https://doi.org/10.1016/j.clineuro.2020.10611 2 (PMID: 32738585; PMCID: PMC7382923)

40. Usman AA, Han J, Acker A et al (2020) A Case Series of Devastating intracranial hemorrhage during venovenous extracorporeal membrane oxygenation for COVID-19. J Cardiothorac Vasc Anesth 34(11):3006-3012. https://doi.org/10.1053/j. jvca.2020.07.063

41. Fletcher-Sandersjöö A, Thelin EP, Bartek J Jr, Broman M, Sallisalmi M, Elmi-Terander A, Bellander BM (2018) Incidence, outcome, and predictors of intracranial hemorrhage in adult patients on extracorporeal membrane oxygenation: a systematic and narrative review. Front Neurol 6(9):548. https://doi.org/10.3389/fneur .2018.00548 (PMID: 30034364; PMCID: PMC6043665)

42. Tan YK, Goh C, Leow AST et al (2020) COVID-19 and ischemic stroke: a systematic review and meta-summary of the literature. J Thromb Thrombolysis 50(3):587-595. https://doi.org/10.1007/ s11239-020-02228-y

43. Jain R, Young M, Dogra S et al (2020) COVID-19 related neuroimaging findings: a signal of thromboembolic complications and a strong prognostic marker of poor patient outcome. J Neurol Sci 414:116923. https://doi.org/10.1016/j.jns.2020.116923

44. John S, Hussain SI, Piechowski-Jozwiak B et al (2020) Clinical characteristics and admission patterns of stroke patients during the COVID 19 pandemic: a single center retrospective, observational study from the Abu Dhabi, United Arab Emirates. Clin Neurol Neurosurg 199:106227. https://doi.org/10.1016/j.cline uro.2020.106227

45. Katz JM, Libman RB, Wang JJ, Sanelli P, Filippi CG, Gribko M, Pacia SV, Kuzniecky RI, Najjar S, Azhar S (2020) Cerebrovascular complications of COVID-19. Stroke 51(9):e227-e231. https:// doi.org/10.1161/STROKEAHA.120.031265 (PMID: 32757751; PMCID: PMC7467046)

46. Shahjouei S, Naderi S, Li J, Khan A, Chaudhary D et al (2020) Risk of stroke in hospitalized SARS-CoV-2 infected patients: a multinational study. EBioMedicine. 59:102939. https://doi. org/10.1016/j.ebiom.2020.102939 (PMID: 32818804; PMCID: PMC7429203)

47. Siegler JE, Cardona P, Arenillas JF et al (2020) Cerebrovascular events and outcomes in hospitalized patients with COVID-19: the 
SVIN COVID-19 multinational registry. Int J Stroke. https://doi. org/10.1177/1747493020959216

48. Yoon BC, Buch K, Lang M, Applewhite BP, Li MD, Mehan WA Jr, Leslie-Mazwi TM, Rincon SP (2020) Clinical and neuroimaging correlation in patients with COVID-19. Am J Neuroradiol 41(10):1791-1796. https://doi.org/10.3174/ajnr.A6717 (PMID: 32912875; PMCID: PMC7661080)
49. Pavlov V, Beylerli O, Gareev I, Torres Solis LF, Solís Herrera A, Aliev G (2020) COVID-19-related intracerebral hemorrhage. Front Aging Neurosci 12:600172. https://doi.org/10.3389/fnagi .2020.600172

50. Perry R, Banaras A, Werring DJ et al (2020) What has caused the fall in stroke admissions during the COVID-19 pandemic? J Neurol 267:3457-3458. https://doi.org/10.1007/s00415-020-10030-2 